\title{
论 文
}

\section{生物活性玻璃-胶原-磷酸丝氨酸复合支架的调控矿 化和生物反应}

\author{
杨春蓉 $^{(1)}$, 王迎军 ${ }^{2}$, 陈晓峰 ${ }^{2}$ \\ (1) 福建工程学院材料科学与工程系, 福州 350108; \\ (2) 华南理工大学材料科学与工程学院, 特种功能材料教育部重点实验室, 广州 510640 \\ E-mail: 1ambmeo@163.com
}

收稿日期: 2011-10-03; 接受日期: 2012-02-04

福建省教育厅科技计划(批准号: JK2009021)资助项目

\begin{abstract}
摘要应用组织工程的策略制备仿生复合支架是修复骨组织缺损的一种新兴方法. 本研究通 过混合交联生物活性玻璃、胶原和磷酸丝氨酸, 制备了一种新型的仿生复合支架. 该支架具有 与天然骨组织相类似的成分. 体外实验表明, 复合支架在不含 MC3T3-E1 细胞的培养液中矿化 产生了八面体晶体, 与复合支架和细胞共同培养过程中的矿化产物在形态和成分上一致. 这种 八面体晶体最后演变成板状差基磷灰石. 支架的细胞外基质类似组分对矿化具有明显的调控作 用. 体内动物实验证实, 该复合支架具有优良的骨修复性能.
\end{abstract}

关键词

复合

支架

细胞

生物矿化

调控
具有特定骨修复功能的复合材料的设计是骨组 织工程研究的热点, 并已在许多方面取得了重大进 展 $^{[1 \sim 3]}$. 很多材料对于某些类型的细胞是生物相容的, 但为了提高其对细胞的反应, 往往需要添加生长因 子和其他试剂. 这就导致了制备方法复杂、成本

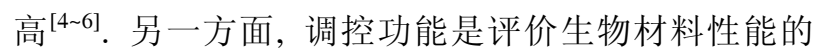
基本因素. 生物材料对细胞的调控作用直接影响着 细胞的黏附、增殖、生长以及随后的生物矿化和骨重 塑效果 ${ }^{[7 \sim 9]}$.

通过对骨组织结构和成分的仿生研究发现, 基 质为矿化反应提供了一个适宜的微环境. 硬组织的 细胞外基质(ECM) 由有机相和无机相组成. 无机相的 主要成分是羟基磷灰石(HA), 有机相包括胶原蛋白 (COL) 和少量非胶原蛋白, 如多糖、脂类等. 这类非 胶原蛋白在骨组织中虽然含量少, 却具有独特的分 布和生物学功能. 例如磷酸丝氨酸 (phosphoserine,
PS), 它是细胞生物膜结构的重要组成成分, 为细胞 分化、增殖和再生所必需, 可维持和促进多种膜结合 蛋白和受体的生物活性. 另外, 它作为磷蛋白的重要 组成成分, 是生物矿化的重要调节剂 ${ }^{[10]}$. 自然衍生的 胶原作为天然骨基质的主要有机成分, 具有良好的 细胞亲和性, 可与细胞发生特定的相互反应 ${ }^{[1]}$. 硅酸 盐和磷酸盐系统的玻璃以及玻璃陶瓷具有良好的生物 相容性, 无毒副作用, 且由于它们的化学组成与生物 体的自然骨骼相似, 因此容易与周围骨骼形成紧密牢 固的化学键合, 或经生物降解形成新的骨骼成分 ${ }^{[12]}$.

本研究旨在优化综合生物活性玻璃(BG)、胶原和 磷酸丝氨酸的生物降解性和生物活性, 设计一种仿 生型骨组织工程支架材料, 重点研究复合材料的生 物分子, 包括胶原蛋白和磷酸丝氨酸对矿化的调控 功能. 这种研究策略的主要优点在于其制备过程简 单、成本低.

英文版见: Yang C R, Wang Y J, Chen X F. Mineralization regulation and biological influence of bioactive glass-collagen-phosphatidylserine composite scaffolds. Sci China Life Sci, 2012, 55, doi: 10.1007/s11427-012-4291-1 


\section{1 材料与方法}

\section{1 复合支架的制备}

$58 \mathrm{~S}$ 生物活性玻璃为 $\mathrm{SiO}_{2}-\mathrm{CaO}-\mathrm{P}_{2} \mathrm{O}_{5}$ 三元系统, 由溶胶-凝胶法制备. 其化学组成为 $(\mathrm{wt} \%)$ : $60 \% \mathrm{SiO}_{2}$, $36 \% \mathrm{CaO}$ 和 $4 \% \mathrm{P}_{2} \mathrm{O}_{5}$.

本实验主要采用冷冻干燥和化学交联技术制备 BG-COL-PS 复合支架. 首先, 将 I 型胶原蛋白溶液 和磷酸丝氨酸按 $4: 1$ 的质量比混合, 摚拌均匀. 随后, 将生物活性玻璃粉体缓慢加入到上述溶液中, 搅拌 均匀. 其中, 生物活性玻璃与有机成分的质量比为 65:35. 之后, 以 1-乙基-3-(3-二甲基氨丙基)碳二亚 胺(EDC)和 $\mathrm{N}$-羊基琥珀酰亚胺(NHS)对上述混合物进 行交联处理. 以乙撑磺酸(MES)为缓冲剂, 调 $\mathrm{pH}$ 至 5.5. 将混合物于 $4^{\circ} \mathrm{C}$ 放置 $24 \mathrm{~h}$ 进行交联反应. 之后, 将上述交联过的混合溶液置于 $-60^{\circ} \mathrm{C}$ 冰箱冷冻 $12 \mathrm{~h}$. 最后冷冻干燥得最终样品.

DMEM 培养液和胎牛血清购自美国密苏里州圣 路易斯 Sigma 公司, 组织培养器血购自美国密歇根州 米德兰 Corning 公司. 小鼠成骨前体细胞株(MC3T3E1)购自日本 RIKEN 细胞库. 其他试剂均为分析纯.

\section{2 矿化实验}

当 MC3T3-E1 细胞培养到足够多时，加入 $0.25 \%$ 胰酶进行消化, 1 2 min 后弃除胰酶, 加入培养液中 止消化, 冲下贴壁的成骨细胞, 吹打均匀制成细胞悬 液, 并将细胞悬液密度稀释成 $1.0 \times 10^{4}$ 个 $/ \mathrm{mL}$. 之后, 取 $2 \mathrm{~mL}$ 细胞悬液接种于放置有 BG-COL-PS 复合支 架的培养板中. 在培养箱中静置, 使细胞完全贴壁于 材料. 培养箱温度设定为 $37^{\circ} \mathrm{C}$, 并保持浓度为 $5 \%$ 的 $\mathrm{CO}_{2}$ 气氛.

材料在含细胞的培养液中分别培养 1,7 和 15 天 后取出, 浸入 PBS 中, 漂洗材料及细胞表面. 然后将 材料放入青霉素小瓶中, 加入 $3 \%$ 龙二醛, 在 $4{ }^{\circ} \mathrm{C}$ 固定 $1 \mathrm{~h}$. 随后吸出固定液, 用 PBS 清洗 2 次. 之后, 用系 列梯度乙醇 $(30 \%, 50 \%, 70 \%, 90 \%, 95 \%$ 和 100\%)脱水, 每种浓度的乙醇通过 2 次, 每次 $5 \mathrm{~min}$. 随后将样品 放入冷冻干燥机中, 抽真空. 样品干燥后取出. 最后, 将样品在铝座上固定, 在 $15 \mathrm{~mA}$ 的电流条件下喷金 3 min, 并用扫描电子显微镜(30XLFEG 型, 荷兰 Phil- ips 公司)和能谱仪(INCA 型, 英国 Oxford 公司)进行 观察. 此外, 本实验还采用 $\mathrm{X}$ 射线衍射仪 $\left(X^{\prime}\right.$ PertPRO 型，荷兰 PANalytical 公司)分析测定支架材料矿化产 物的晶相结构. $\mathrm{X}$ 射线衍射仪采用 $\mathrm{Cu}$ 靶, $\lambda$ 为 0.15418 , 管压为 $40 \mathrm{keV}$, 对样品从 $10^{\circ} \sim 70^{\circ}$ 进行 $2 \theta$ 扫描, 扫描 速度为 $15.24 \% \mathrm{~min}$.

\section{3 骨缺损动物模型制备}

选用新西兰大白兔(2 2.5 kg，12 月龄)考察复合 材料的骨组织反应. 对每只兔静脉注射布托啡诺酒 石酸 $(0.2 \mathrm{mg} / \mathrm{kg})$ 和咪唑安定 $(0.02 \mathrm{mg} / \mathrm{kg})$, 并在 $2 \%$ 异 氟醚的气氛下按 $3 \mathrm{mg} / \mathrm{kg}$ 的剂量注射异丙酚进行麻醉. 之后，每只兔随机选用左右侧桡骨做 $20 \mathrm{~mm}$ 切口的 骨缺损，植入尺寸为 $15 \mathrm{~mm} \times 20 \mathrm{~mm}$ 的 BG-COL-PS 支架材料. 空白对照组不植入任何物质. 用肌肉覆盖 缺损区，缝合皮肤. 术后 12 周取材，石蜡包埋切片， 并用苏木精伊红(HE)染色, 于光学显微镜下观察成 骨以及材料降解情况

\section{2 结果与讨论}

\subsection{MC3T3-E1 细胞与 BG-COL-PS 复合支架的 生物矿化}

相差显微镜观察发现，成骨细胞与 BG-COL-PS 复合支架材料共同培养 7 天后有机结合在一起, 形成 一个由细胞和矿化细胞间质组成的三维晶体结构, 如图 1A 所示. 通过扫描电子显微镜及其能谱对这种 矿物进行分析, 可以发现矿物呈规则八面体结构, 含 大量钙离子, 如表 1 和图 2 所示. 由细胞分泌所形成 的八面体晶体在形成矿物的密度及形状之后, 细胞 分泌过程产生新的晶体单元, 矿物的沉析作用处于 一个动态的不断更新的运动之中. 晶体宏观上的生 长通过大量生长单元的组装堆砌而实现, 如图 1B D 所示.

为进一步证实 BG-COL-PS 复合支架材料的调控 机制，比较了这种复合材料在不含有细胞培养液中 的矿化情况. 结果发现, 培养 7 天后, 材料表面同样 生成八面体晶体(图 3), 与细胞和材料共同调控矿化 7 天产生的晶体形貌几乎一样. 能谱元素分析表明, 这两种晶体具有相同的元素及相近的组成(表 1 和 2). 随后，这种晶体进一步熔融生长, 15 天后演变成板状 
矿物(图 4).

矿化晶体的形态变化表明, BG-COL-PS 复合支 架材料对晶体的生长有着极大的调控作用, 可诱导 矿化并使晶体生长成特定的形态. 在本实验的矿化 系统中, 晶体在材料上的沉积似乎影响了胶原和磷 酸丝氨酸基质的熔融(图 3), 而这种基质的熔融反过 来又加速了晶体的自组装.

根据热力学第二定律, 胶原和磷酸丝氨酸基质
在溶液中的矿化反应使得局部自由能达到最小. 结 构的进化需要系统熵的降低, 如果没有这些相互反 应造成的最小自由能，熵值将会使形成的晶体具有 更低水平的组装形式, 以更简单的不规则的形貌出 现, 而非图 2 所观察到的规则的八面体状矿物晶体. 这种能量顺序的影响一旦出现, 可使亚单元小分子 自组装成具有特定形貌的宏观矿物, 从而加速骨缺 损的修复.

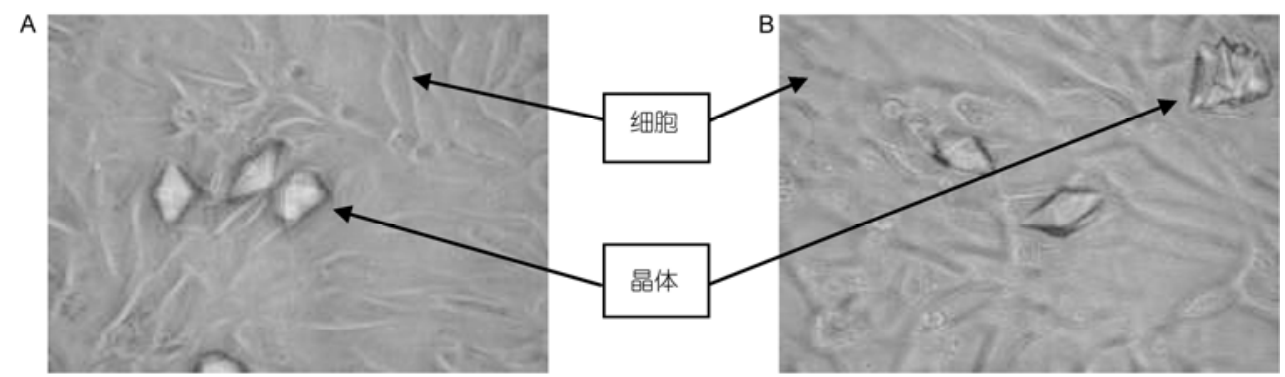

C

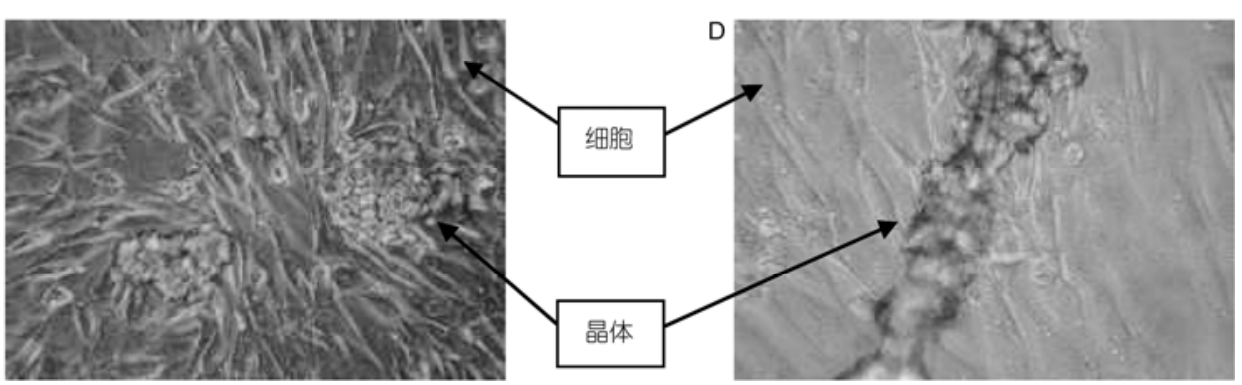

图 1 BG-COL-PS 支架材料与细胞培养 7(A), 8(B), 10(C) 和 12(D)天调控矿化显微镜照片 $(\times 200)$

表 1 BG-COL-PS 支架材料与细胞培养 7 天矿化晶体 EDX 分析

\begin{tabular}{cccc}
\hline 元素 & $K$ 比率 & 质量百分比 $(\%)$ & 原子量百分比 $(\%)$ \\
$\mathrm{C}$ & 0.1404 & 14.037 & 33.629 \\
$\mathrm{O}$ & 0.1929 & 19.285 & 34.685 \\
$\mathrm{P}$ & 0.0084 & 0.842 & 0.783 \\
$\mathrm{Au}$ & 0.2861 & 28.612 & 4.180 \\
$\mathrm{Ca}$ & 0.3722 & 37.224 & 26.724 \\
总计 & & 100.000 & 100.000 \\
\hline
\end{tabular}

表 2 BG-COL-PS 支架材料在不含细胞的培养液中培养 7 天矿化晶体 EDX 分析

\begin{tabular}{ccrr}
\hline 元素 & $K$ 比率 & 质量百分比 $(\%)$ & 原子量百分比 $(\%)$ \\
$\mathrm{C}$ & 0.1312 & 13.121 & 34.556 \\
$\mathrm{O}$ & 0.1816 & 18.160 & 35.904 \\
$\mathrm{P}$ & 0.0171 & 1.709 & 1.746 \\
$\mathrm{Au}$ & 0.3991 & 39.916 & 6.510 \\
$\mathrm{Ca}$ & 0.2710 & 27.094 & 21.384 \\
总计 & & 100.000 & 100.000 \\
\hline
\end{tabular}




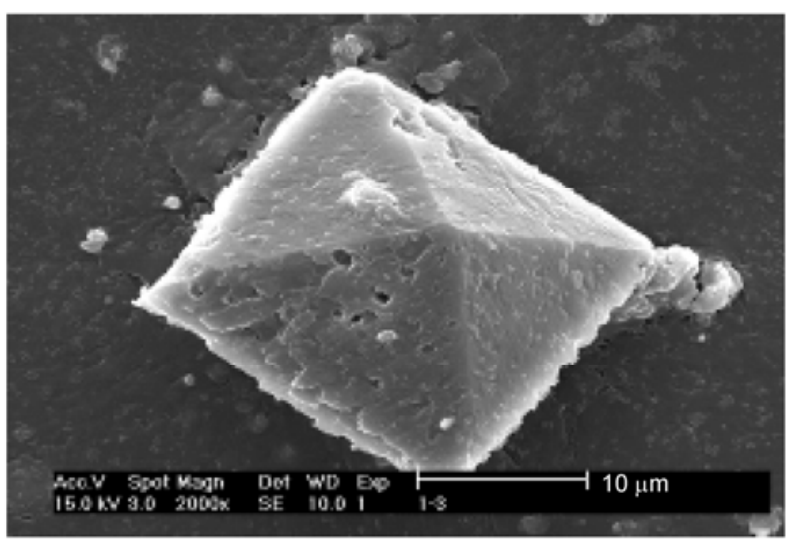

图 2 BG-COL-PS 支架材料与细胞在培养液中培养 7 天 矿化晶体扫描电子显微镜形貌观察

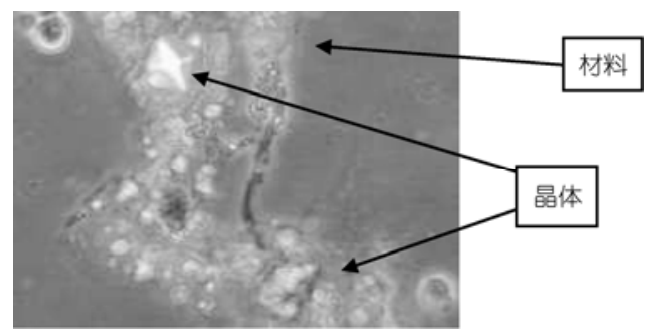

图 3 BG-COL-PS 支架材料在不含细胞的培养液中培养 7 天矿化光学显微镜照片 $(\times 200)$

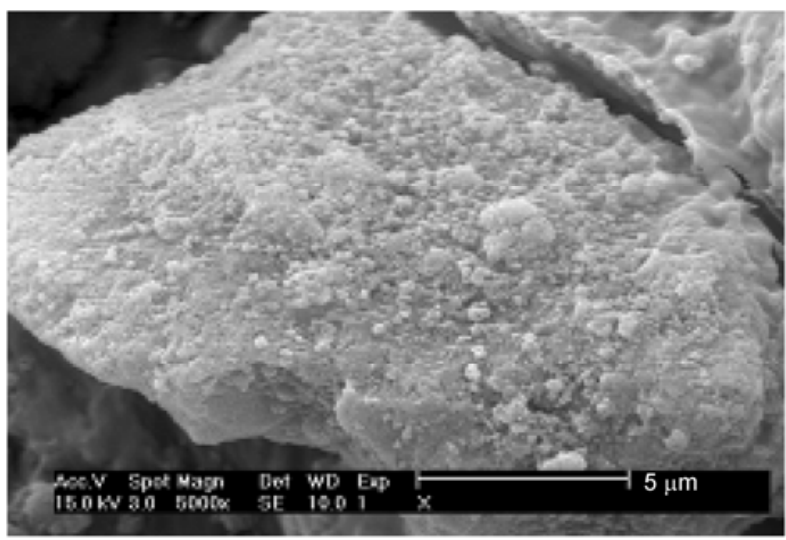

图 4 BG-COL-PS 支架材料在不含细胞的培养液中培养 15 天矿化晶体扫描电子显微镜形貌观察

采用 XRD 对 BG-COL-PS 复合支架调控矿化 15 天生成的晶体进行分析, 如图 5 所示. 由图谱可知, 矿化物为羟基磷灰石. 但与标准羟基磷灰石陶瓷粉 末的衍射峰相比, 矿化物的衍射峰明显变宽, 峰位重 叠, 表明矿物相的生长能减少, 结晶度较低, 晶粒细 小, 该特点与天然骨很类似 ${ }^{[12]}$.

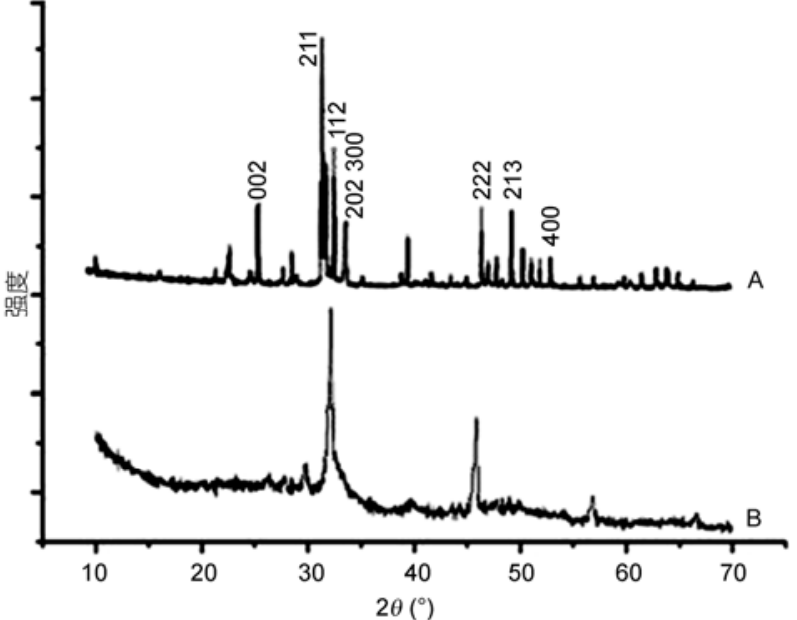

图 5 羟基磷灰石标准样品(A)和 BG-COL-PS 支架材料(B) 在不含细胞的培养液中培养 15 天矿化晶体 XRD 图

羟基磷灰石在不含细胞的培养液中的形成证明, BG-COL-PS 生物活性骨组织工程支架具有显著的引 导和调控矿化的能力. 这种能力对于骨修复和骨组 织工程材料极其重要. 许多材料对于某些类型的细 胞是生物相容的, 但为了提高其对细胞的反应, 往往 需要添加生长因子和其他试剂. 本研究表明, 当不添 加任何额外的细胞和试剂时, 产生了类似的细胞反 应，如差基磷灰石的形成.

在本实验的两种矿化反应系统中, 钲和磷的浓 度变化不大, 因此复合支架中的生物分子, 如胶原蛋 白和磷酸丝氨酸可能是调控羟基磷灰石矿化的关键 因素.

在自然骨组织中, 生物分子调控是影响生物矿 化的基本因素 ${ }^{[13]}$. 生物分子内的羧基(-COOH)和磷 酸基团 $\left(-\mathrm{PO}_{4} \mathrm{H}_{2}\right)$ 对钻离子有很强的亲和力, 可促进和 调控矿化. 胶原在生物矿化的初始阶段起着重要作 用. 但胶原本身不能完成基质的全部功能. 它不能引 起和指导矿化，起这些作用的是磷蛋白. 磷蛋白是一 类富含天冬氨酸及磷酸丝氨酸的蛋白质. 不同的生 物体系可能采用不同的矿化基质，但是所有矿化基 质的共性都是通过磷酸基与 $\mathrm{Ca}^{2+}$, 进而与磷酸钙微 晶结合. 磷酸丝氨酸的另一重要特征是它能与胶原 产生较强的结合. 磷酸丝氨酸的羧基和氨基可以与 胶原的翔基和氨基分别发生键合作用，这样的配合 为磷酸钻的析出与组装创造了条件.

本实验制备的 BG-COL-PS 复合支架正是由于具 
有胶原和磷酸丝氨酸这类天然细胞外基质成分，因 此可起到类似细胞分泌的细胞外基质的模板作用而 完成调控矿化的过程, 形成羟基磷灰石.

\section{2 组织学评价}

图 6 为 BG-COL-PS 支架材料植入骨缺损 12 周 时的 HE 染色切片组织学形貌. 由图可见, 术后 12 周, 缺损处的材料周围形成了具有哈弗氏系统的新骨. 在新形成的骨组织中, 还可发现逐渐钙化的软组织. 材料与骨组织间紧密联接.

由此可见，该材料参与了体内的骨重建过程. 一 般而言，体内的骨重建过程往往由破骨细胞的吞噬 作用以及成骨细胞的成骨作用在自体骨植入材料周 围发生. 有些材料尽管可在体内和体外被破骨细胞 吸收 ${ }^{[14 ~ 16]}$, 但由于这些材料的吸收和溶解速率太慢, 因此很难将其应用于骨缺损的修复. BG-COL-PS 复 合材料由生物活性玻璃晶体和细胞外基质成分, 如胶 原蛋白和磷酸丝氨酸组成, 因此易于被黏附的细胞吸 收，在体液中易于溶解，产生明显的骨重塑效果.

\section{3 结论}

BG-COL-PS 复合支架对矿化的调控功能及其生 物反应的研究表明，在不含细胞的培养液中，该支架 可以矿化生成八面体晶体，与支架和细胞共同调控 矿化的产物在形态和成分上相类似。这一调控功能

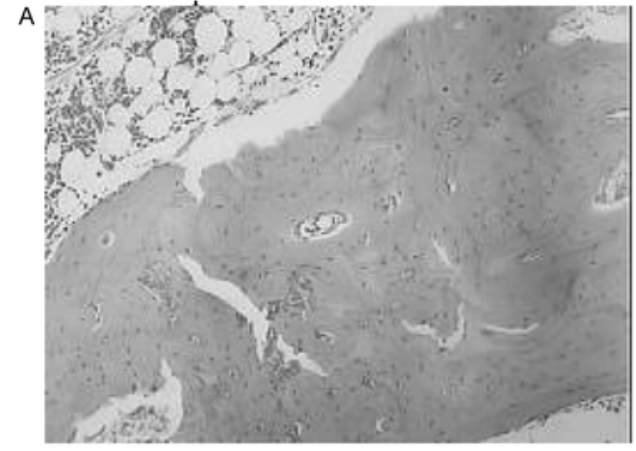

B

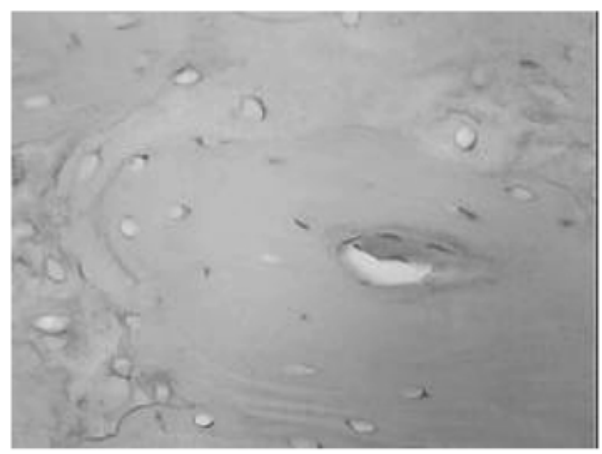

图 6 BG-COL-PS 复合支架植入骨缺损 12 周组织学形貌 观察

A: $\times 10 ; B: \times 40$

主要受复合材料中有机质成分影响. 而且, 具有高生 物活性的 BG-COL-PS 复合支架在体内表现出优良的 骨修复效果.

\section{参考文献}

1 Dalby M J, Childs S, Riehle M O. Fibroblast reaction to island topography: changes in cytoskleton and morphology with time. Biomaterials, 2003, 24: 927-935

2 Palmer L C, Newcomb C J, Kaltz S R. Biomimetic systems for hydroapatite mineralization inspired by bone and enamel. Chem Rev, 2008, 108: 4754-4783

3 Dalby M J, Riehle M O, Johnstone H. In vitro reaction of endothelial cells to polymer demixed nanotopography. Biomaterials, 2002, 23: 2945-2954

4 Barbucci R, Magnani A, Lamponi S. The use of hyaluronan and its sulphated derivative patterned with micrometric scale on glass substrate in melanocyte cell behaviour. Biomaterials, 2003, 24: 915-926

5 王桢，张正，张俊川，等. 体积较大的多孔聚酯支架中的骨髓基质干细胞的分布. 科学通报, 2009, 54: 1207-1213

6 吴涛, 南开辉, 陈景帝, 等. 可控释淫羊蕉苷的仿生组装骨诱导修复材料. 科学通报, 2009, 54: 1198-1206

7 Silver I A, Deas J, Erecinska M. Interactions of bioactive glasses with osteoblasts in vitro: effects of 45S5 Bioglass, and 58S and 77S bioactive glasses on metabolism, intracellular ion concentrations and cell viability. Biomaterials, 2001, 22: 175-185

8 张举成, 刘卫, 易中周, 等. 竹红菌乙素光动力作用调控胶原蛋白中吡啶啉交联含量: 光动力治疗纤维化症机制的初探. 科学通报, 2009, 54: 2076-2081

9 Xynos I D, Hukkanen M V J, Batten J J. Gene expression profiling of human osteoblasts following treatment with the ionic products of Bioglass 45S5 dissolution. Calcif Tissue Int, 2000, 67: 321-329

10 Varma H K, Yokogawa Y, Espinosa F F, et al. Porous calcium phosphate coating over phosphorylated chitosan film by a biomimetic 
method. Biomaterials, 1999, 20: 879-884

11 Cui F Z, Wang Y, Zhang W. Conformation change of collagen during the initial stage of biomineralization of calcium phosphate. J Mater Sci-Mater, 2008, 18: 35-38

12 Hench L L. Bioceramics. J Am Ceram Soc, 1998, 81: 1705-1728

13 Place E S, Evans N D, Stevens M M. Complexity in biomaterials for tissue engineering. Nat Mater, 2009, 8: 457-470

14 Rafat M, Li F, Fagerholm P. PEG-stabilized carbodiimide crosslinked collagen- chitosan hydrogels for corneal tissue engineering. Biomaterials, 2008, 29: 3960-3972

15 Cao H, Xu S Y. EDC/NHS-crosslinked type II collagen-chondroitin sulfate scaffold: characterization and in vitro evaluation. J Mater Sci Mater Med, 2008, 19: 567-575

16 Wang Y J, Yang C R, Chen X F. Investigation of the porous biomaterials for bone reconstruction with addition of biomimetic nano-sized inorganic particles. Key Eng Mater, 2007, 336-338: 1534-1537 